\title{
Tumour stem cells generate vasculature
}

Glioblastoma is a highly angiogenic tumour and, although it is known that neural stem cells closely interact with the vascular niche, the origins of the endothelial cells in this malignancy are poorly understood. Two new papers show that stem-like cells in the tumour can differentiate into endothelial cells, thereby generating the tumour vasculature.

Both Wang et al. and Ricci-Vitiani et al. began by examining the angiogenic endothelium in glioblastoma tissue from patients, using a range of endothelial and glioblastoma markers. Both groups found that a proportion of the endothelial cells had the same genomic alterations as the tumour cells, indicating that they were of neoplastic origin.

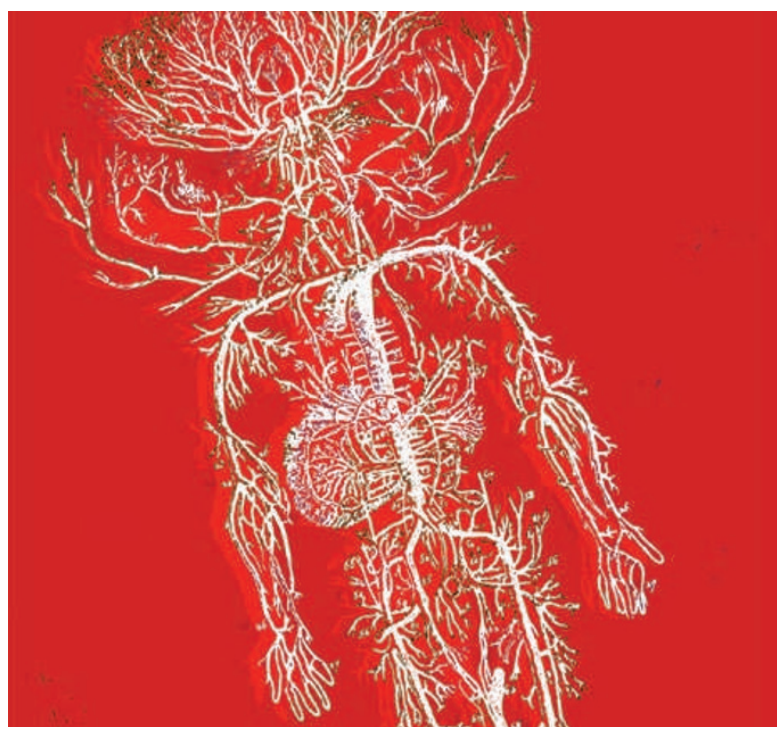

Wang et al. focused on the $\mathrm{CD} 133^{+}$stem cell-like fraction of tumour cells and found a subset of cells expressing vascular endothelial cadherin (also known as CD144) that behaved like endothelial progenitors. These cells could mature into endothelial cells. Lineage analyses and single-cell clonal studies showed that a subpopulation of this $\mathrm{CD} 133^{+}$stem cell-like fraction is multipotent, differentiating into both tumour and endothelial cell types. Many of the cells from these fractionated populations also had the genomic aberrations seen in the parent tumour. Importantly, blocking vascular endothelial growth factor A pharmacologically with bevacizumab (Avastin; Genentech/ Roche) or using small hairpin RNAs did not inhibit the differentiation of $\mathrm{CD}_{133^{+}}$cells into endothelial cell types.

When $\mathrm{CD} 133^{+} \mathrm{CD} 144^{-}$or $\mathrm{CD} 133^{+} \mathrm{CD} 144^{+}$cells were injected into immunodeficient mice, large, highly infiltrative tumours developed, with the tumours from $\mathrm{CD} 133^{+} \mathrm{CD} 144^{+}$showing the highest levels of vascularization. The blood vessels in these tumours expressed human markers, confirming that they had developed from the xenografted cells.

Ricci-Vitiani et al. examined CD $31^{+} \mathrm{CD} 144^{+}$endothelial cells from human glioblastomas - CD31 is another protein that is expressed on endothelial cells. They found that many of the cells expressing CD31 and CD144 had the same genomic alterations as the tumour cells, and some cells co-expressed glial and mature endothelial cell markers. When the $\mathrm{CD} 31^{+} \mathrm{CD} 144^{+}$cells were injected into immunodeficient mice, a subset of these cells produced highly vascularized, undifferentiated tumours. Undifferentiated cell populations from human glioblastoma tissue also formed vascularized tumours in immunodeficient mice, and a high percentage of the endothelial cells in the vessels in the middle of these tumours were of human origin. When these glioblastoma-derived endothelial cells in the xenografts were selectively killed, the tumours decreased in size and became necrotic, showing that vessels containing these cells are essential for the survival of specific regions of the tumour.

The findings of these two research groups describe a novel link between glioblastoma cells and endothelial progenitors and thus, a new mechanism for tumour vascularization. This work may inform development of therapeutic strategies for this aggressive malignancy.

Ezzie Hutchinson

ORIGINAL RESEARCH PAPERS Wang, R. et al.

Glioblastoma stem-cell like cells give rise to tumour endothelium. Nature 21 Nov 2010 (doi:10.1038/nature09624)|Ricci-Vitiani, L. et al. Tumour vascularization via endothelial differentiation of glioblastoma stem-like cells. Nature 21 Nov 2010 (doi:10.1038/nature09557) 